from the same time period with the same genotype were included in the phylogenomic analysis. The genomic variants identified by WGS were used for phylogenomic inference, drug resistance prediction and to determine genomic distances between isolates.

WGS analysis revealed unexpected genomic diversity within the seemingly homogenous IS 6110 cluster of $M$. tuberculosis isolates. Despite the IS6110 RFLP based uniformity, at least six non-time dependent sub-clusters and several orphan-isolates were evident from the WGS-based phylogeny and genomic comparisons. Sub-clusters gained drug resistance conferring mutations (beyond MDR) on multiple occasions and $M$. tuberculosis isolates from surrounding suburbs were observed throughout the phylogeny.

IS6110 RFLP typing underestimated the complexity of this 23-year outbreak. This study suggests that there is continuous circulation and reintroduction of this $M$. $t u$ berculosis cluster in the community setting. Even with the advent of the WGS-era, confirming direct epidemiological links or outbreak directionality remains a challenge in high TB burden, low-income settings.

6.13

doi: 10.15789/2220-7619-2018-4-6.13

\section{A 15-YEAR SPATIOTEMPORAL ANALYSIS OF MYCOBACTERIUM TUBERCULOSIS LINEAGES 1 AND 2 IN CHIANG RAI, THAILAND}

A. Disratthakit ${ }^{1}$, P. Palittapongarnpim ${ }^{2,3}$, P. Ajawatanawong ${ }^{2}$, N. Smittipat ${ }^{3}$, S. Mahasirimongkol ${ }^{1}$, R. Miyahara ${ }^{4}$, H. Yanai ${ }^{5,6}$, N. Yamada ${ }^{7}$, S. Nedsuwan ${ }^{8}$, W. Imasanguan ${ }^{8}$, P. Kantipong ${ }^{8}$, B. Chaiyasirinroje ${ }^{5}$, S. Bupachat ${ }^{5}$, P. Ananpradit ${ }^{5}$, W. Ruengchai ${ }^{2}$, T. Juthayothin ${ }^{3}$, J. Phelan', J. Parkhill ${ }^{10}$, T.G. Clark ${ }^{9}$, M.L. Hibberd ${ }^{9}$, K. Tokunaga ${ }^{4}$ ${ }^{1}$ Department of Medical Sciences, Ministry of Public Health, Thailand; ${ }^{2}$ Department of Microbiology, Faculty of Science, Mahidol University, Thailand; ${ }^{3}$ National Centre for Genetic Engineering and Biotechnology, National Science and Technology Development Agency, Thailand; ${ }^{4}$ Department of Human Genetics, Graduate School of Medicine, the University of Tokyo, Japan; ${ }^{5}$ TB-HIV Research Foundation, Thailand; ${ }^{6}$ Fukujuji Hospital, Japan Antituberculosis Association (JATA), Japan; ${ }^{7}$ Research Institute of Tuberculosis, JATA, Japan; ${ }^{8}$ Chiangrai Prachanukroh Hospital, Ministry of Public Health, Thailand; ${ }^{9}$ London School of Hygiene and Tropical Medicine, United Kingdom; ${ }^{10}$ Welcome Trust Sanger Institute, Hinxton, United Kingdom

Chiang Rai is the northernmost province of Thailand with high burden of tuberculosis (TB) and high TB death rate. Chiang Rai consists of various ethnic groups in three different geographic areas including (1) the bordering area in the northern and northeastern, (2) the central area, and (3) the outlying districts in the southeastern, southern and southwestern.

We aimed to assess the spatial and temporal distribution of Mycobacterium tuberculosis (MTB) lineages in the three different areas over 15 years.

Whole genome sequence (WGS) data was used to classify the genotypes of 1497 MTB using lineage-specific single nucleotide polymorphisms (SNPs). The spatiotemporal distribution of MTB lineage was analyzed in the three different areas during early 2000s (2002-2006), late 2000s (2007-2011) and 2010s (2014-2018). Stoddart and Taylor's index $(G)$ was calculated to determine genotypic diversity of MTB lineage in the different settings.

In 2000s, lineage 2 (East Asian) was a highly predominant genotype (45\%) in Chiang Rai followed by lineage 1 (Indo-oceanic) (41\%). In 2010s, lineage 1 became the most dominant genotype $(51 \%)$ replacing lineage 2 (35\%).
The overall change in predominant lineage from lineage 2 to lineage 1 was caused by a dramatic increase proportion of lineage 1 in the central area (from 44 to $53 \%$ ) and in the outlying districts (from 39 to 59\%). In the bordering area, a combined impact of increasing distribution of lineage 1 (from 32 to $40 \%$ ) and other lineages (from 18 to 24\%) was an additional cause of changing predominant lineage. The Stoddart and Tayloy's analysis of genotypic diversity showed that the central area had the highest diversity of MTB lineage $(\mathrm{G}=4.14 \pm 0.46)$ followed by the bordering area $(\mathrm{G}=3.67 \pm 0.76)$ and the outlying districts $(\mathrm{G}=$ $3.63 \pm 0.62)$.

Our study combining genotypic and space-time analysis has revealed a dynamic population changes in MTB lineage over 15-year period in Chiang Rai. Further studies on social determinants and patient's demographic data in the different geographic areas have the potential to provide effective TB control in the different setting.

6.14 doi: 10.15789/2220-7619-2018-4-6.14

\section{MOLECULAR-GENETIC METHODS OF DETECTION OF TUBERCULOSIS AND ITS DRUG RESISTANCE IN ARKHANGELSK REGION IN 2017}

\section{P.I. Eliseev ${ }^{1}$, I.V. Tarasova ${ }^{2}$, A.O. Mariandyshev ${ }^{1}$}

${ }^{I}$ Northern State Medical University, Arkhangelsk, Russia; ${ }^{2}$ Arkhangelsk Regional Antituberculosis Dispensary, Arkhangelsk, Russia

Burden of tuberculosis (TB) is decreasing in Arkhangelsk region in northwestern Russia with incidence declining from 45.9/100 000 in 2012 to 21.6/100 000 in 2017 in civil society (excluding penitentiary society). Introduction of molecular-genetic tests of detection of TB and its drug resistance (DR), including multidrug-resistant (MDR) and extensively drug resistant (XDR) TB, is one of the key components of regional TB program. It plays an important role in improvement of diagnostics and management of TB patients in the region.

The objective was to evaluate performance of molecular-genetic tests used for detection of TB and its DR among patients with TB registered in civil society in Arkhangelsk region in 2017.

Line probe assay (LPA) (Hain Lifescience, Germany) and GeneXpert MTB/RIF (Cepheid, USA) were used for detection of $M$. tuberculosis (MTB) and its DR alongside conventional sputum smear microscopy (SM) and culture. All patients were initially tested with SM followed by LPA (Genotype MTBDRplus) if result of SM was positive or GeneXpert if result was negative using the same sample. In case of DR to isoniazid and/or rifampicin, Genotype MTBDR $s l$ was performed to detect additional DR to fluoroquinolones and injectables, including XDR. In cases suggestive of nontuberculous mycobacteria (NTM), SM or culture positive with negative results of LPA or GeneXpert for MBT, identification was performed using Genotype Mycobacterium CM/AS.

Total of 214 "new cases" and 28 "relapses" of TB were registered in Arkhangelsk region in 2017. MTB was detected in $160(74.8 \%)$ out of 214 "new cases" and all of them were tested for DR using molecular-genetic tests. 53 patients $(31.1 \%)$ had MDR-TB, among them 3 patients had additional DR to injectables and 2 patients had XDR-TB. Among 28 "relapses" MTB was detected in 24 (85.7\%) patients. 13 patients (54.2\%) had MDR-TB, among them in 1 patient additional DR to injectables and in 2 patients to fluoroquinolones was detected. NTM associated disease was diagnosed in 4 patients $(2-M$. avium, $1-M$. gordonae, $1-M$. interjectum) 
All TB patients in Arkhangelsk region were tested with molecular-genetic tests before the treatment enabling quicker diagnostics and earlier treatment initiation. Early diagnosis ensures proper treatment regimen for patients with TB and NTM diseases. As a result management of TB patients is improved leading to better treatment outcomes and subsequently reduced TB transmission in the region.

\subsection{5}

doi: 10.15789/2220-7619-2018-4-6.15

\section{DETECTION OF EXTRACELLULAR MYCOBACTERIUM TUBERCULOSIS SMALL RNAS}

M.V. Fursov, T.I. Kombarova, I.A. Dyatlov, V.D. Potapov

State Research Center for Applied Microbiology and Biotechnology, Obolensk, Russia

According to WHO, tuberculosis infection is one of the top ten deadly infections in the world, and about one-fourth of the human population is a carrier of latent tuberculosis infection (LTBI) without manifestation of disease symptoms. The LTBI associated with the conversion of $M$. tuberculosis bacilli to a dormant, metabolically inactive state; however, the molecular mechanisms of this change is not well studied. In many researches the small RNAs (sRNAs) was proposed as regulators of these processes. The aim of the study was detection of sRNA transcripts in cultural supernatant of $M$. tuberculosis strain $\mathrm{H} 37 \mathrm{Rv}$ and into the blood serum of mice $(\mathrm{C} 57 \mathrm{Bl})$ infected with LTBI.

Mycobacterial cells were grown in Middlebrook $7 \mathrm{H} 9$ containing $10 \% \mathrm{ADC}$ supplement at $37^{\circ} \mathrm{C}$ and harvested at different growth phase for use. The culture of M. tuberculosis was centrifuged at $6000 \mathrm{~g}$ for $20 \mathrm{~min}$ at $4^{\circ} \mathrm{C}$. The supernatant was filtered through $0.22 \mu \mathrm{m}$ filters to remove the remaining bacteria. Bacterial total RNA was extracted from M. tuberculosis cultural supernatant by ExtractRNA reagent (Evrogen, Moscow, Russia), followed by digestion with Turbo DNase-free kit (Ambion, Austin, TX, USA) to remove contaminating DNA. cDNA was synthesized with $1.5 \mu \mathrm{g}$ of total RNA by M-MLV (Evrogen, Moscow, Russia) transcriptase and random hexadeoxynucleotides according to the manufacture's instruction. The quantitative RT-PCR (qPCR) was carried out with the qPCRmix-HS SYBR (Evrogen, Moscow, Russia) and the CFX-96 real-time PCR detection system (BioRad, USA). All primers used in this study were designed using VectorNTI 11 (Invirogen, USA) and GeneRunner software (http://www.generunner.net) and synthesized at Evrogen (Moscow, Russia). LTBI C57B1 mouse model was designed as previously described (Shramko et al., 2010). The certain sRNA transcripts have been detected in $M$. tuberculosis culture supernatant at different growth phases (exponential phase, stationary phase and late-stationary phase) and into the blood serum of mice infected with LTBI. Obtained data allow us to propose M. tuberculosis sRNAs as new markers for LTBI diagnostic in the future.

\section{TB PORTALS PROGRAM: DATA-DRIVEN MULTI- NATIONAL CONSORTIUM AGAINST DRUG-RESISTANT TUBERCULOSIS}

\section{A.E. Gabrielian, A. Rosenthal}

Office of Cyber Infrastructure and Computational Biology, National Institute of Allergy and Infectious Diseases, National Institutes of Health, Rockvill, MD, USA

TB Portals Program (TBPP) is an international initiative that is founded, developed and steered by doctors, researchers, IT specialists and radiologists to combat drug-resistant tuberculosis (DR-TB). The efforts of hospitals and biomedical research institutes from ten countries on three continents are organized and supported by TBPP to actively establish and grow an integrated open-access network of innovative tools and data from real patient cases of TB (tbportals.nid.nih.gov). The TB Portals collect, analyze, standardize, and present anonymized clinical, laboratory, and socioeconomic data, full bacterial genomes, and radiological data (CXR and CT).

The TBPP database currently has more than 1300 published (22 250 total cases), $75 \%$ of which are MDR or XDR-TB. Clinicians supply and validate all patient data. Once validated, the data become published with open access status in accordance with NIH FAIR principles. Importantly, both original and derived (expert-based and computational annotations) data remain patient-centric, i.e. linked to a unique patient identifier. This cornerstone principle enables users to define and analyze cohorts of patients, augmenting OMICS studies with diverse clinical information.

To date the database contains 730 published (1300 total) fully sequenced and annotated Mycobacterium tuberculosis genomes associated with the patient case record. We will highlight several TBPP projects studying 1) genomic signatures for TB relapse and reinfection, and 2) comparative analysis of M. tuberculosis strains isolated from sputum vs. surgically removed parts of lungs.

TBPP assists doctors and researchers in testing and refining their hypotheses with friendly and powerful tools. Starting from genomics and the molecular basis of drug resistance, we will demonstrate how our online tools enable anyone to simultaneously look at clinical, microbiological and radiological evidences in order to (1) search for genomic clues for inconsistencies in existing DR-TB diagnostics and to (2) study common and countries-specific evolutionary patterns of $M$. tuberculosis.

6.17 doi: 10.15789/2220-7619-2018-4-6.17

\section{SIMPLIFYING NGS APPROACHES TO OPTIMIZE TRACING OF TRANSBORDER SPREAD OF MYCOBACTERIUM TUBERCULOSIS}

\section{Garcia de Viedma}

Clinical Microbiology and Infectious diseases Department, Gregorio Marañón University Hospital, Madrid, Spain; CIBER Enfermedades Respiratorias CIBERES, Madrid, Spain

Molecular epidemiology, and more recently genomic epidemiology, based on whole genome sequencing (WGS), improve our understanding about the transmission dynamics of Mycobacterium tuberculosis in a population. However, in many countries, including many of those with a high burden of TB, systematic genomic epidemiology cannot be implemented. Trying to find a solution to this situation, we propose an alternative line of progression, which tries to conciliate the discriminatory power of WGS with the speed, low cost and simplicity of PCR-based approaches. The cost of this shortcut is that it sacrifices the complete knowledge of all the transmission clusters in a population, because it needs to focus on surveying the strains that deserve special attention because they are more actively transmitted, or correspond to high-risk MDR or XDR strains. This short-cut strategy has proved to be efficient to survey actively transmitted strains, to fast track outbreak-strains, to update the presence of high-risk strains in a population or to give an urgent answer to public-health alerts, such as to rule out secondary cases due to the importation of XDR-TB cases. More recently, we are integrating this strategy to optimize the characteriza- 